\title{
The impact of per diem senior pediatric radiologists in an academic setting
}

\author{
Richard I. Markowitz ${ }^{1,2}$ (D) George A. Taylor ${ }^{1,3} \cdot$ Kassa Darge $^{1,2}$ \\ Received: 30 June 2020 / Revised: 14 August 2020 / Accepted: 13 October 2020 / Published online: 17 November 2020 \\ (C) Springer-Verlag GmbH Germany, part of Springer Nature 2020
}

\section{Introduction}

Traditionally, academic radiology departments are staffed by full-time faculty who provide clinical service and resident/ student teaching while pursuing academic and research goals necessary for advancement and promotion. As the clinical workload, number of complicated cases, and demands for $24 / 7$ coverage have increased, there is often less time available for teaching and research. At the same time, senior faculty members no longer want to participate in expanded hours such as weekend, late day and overnight clinical coverage [1]. In some departments, options are available for reducing these less desirable duties, usually with a corresponding reduction in salary. There might even be options for overall reduction of duties by $10 \%$ to $50 \%$. But the policies at different academic institutions are highly variable and sometimes prohibitive of innovative solutions. For many senior academic radiologists, the choice is full-time or no-time, thus forcing premature retirement and therefore a great loss of experience, expertise and mentoring to the less senior members of the department. These trends are especially relevant in pediatric radiology, given declining interest in pediatric radiology as a career choice [2] and subsequent shortage of trained specialists who want an academic career.

Richard I. Markowitz

markowitz@email.chop.edu

1 Department of Radiology, Children's Hospital of Philadelphia, 3400 Civic Center Blvd., Philadelphia, PA 19104, USA

2 Perelman School of Medicine, University of Pennsylvania, Philadelphia, PA, USA

3 Department of Radiology, Boston Children's Hospital, Boston, MA, USA

\section{How the program works}

In our department at the Children's Hospital of Philadelphia, a large academic standalone pediatric hospital affiliated with a university, we recently began an optional program to allow such valuable senior members of the department to fully retire from the university (an independent entity) and continue to work in the hospital department on a flexible per diem basis without the requirement for weekend, late night or overnight duties.

The per diem radiologist is paid only for each work slot covered (usually a weekday 9-h assignment). Scheduling and availability are determined by mutual consent of the per diem radiologist and the scheduler in advance, in keeping with departmental daily needs and individual radiologists' abilities and preferences. Each per diem is scheduled independently and there is no "shared slot" that must be filled. Likewise, there is no minimum or maximum number of days or slots that must be filled. Occasionally extra days are added in case of unforeseen emergencies or changes in the schedule. The per diems do not have funded academic or administrative time. They do participate in the teaching program but have no research responsibilities or requirements.

To see how the per diem program was working, we audited a sample period just prior to the current viral pandemic. During a 6-month period (September 2019 to February 2020), three senior diagnostic pediatric radiologists and one senior pediatric interventional radiologist participated in a per diem employment arrangement. The number of full-day shifts, studies read, relative value units (RVUs) generated, and compensation costs were measured and compared to a typical fulltime equivalent (FTE) faculty member in the Division of Body Imaging. The type of studies read was considered and compared. Because the single per diem interventional radiologist worked only half that time period, these data were not included.

Three per diem radiologists worked a total of 99 daytime weekday slots and read a total of 5,644 diagnostic studies in 
that 6-month period. In comparison, a typical FTE diagnostic radiologist covered 94 clinical weekday slots and read 5,674 studies, which was comparable but included a higher percentage of MRI and CT examinations (Table 1).

The majority of the diagnostic studies read by the per diem group consisted of conventional radiographs (71.5\%). The non-radiograph portion was $28.5 \%$, comprising $20 \%$ ultrasound, $7 \%$ fluoroscopy, $1 \% \mathrm{CT}$ and $0.5 \% \mathrm{MR}$ exams.

The total RVUs generated by the per diem group was 2,052, whereas one typical FTE body radiologist with $10 \%$ academic time (equivalent to 0.9 clinical FTE) generated 2,238 RVUs, reflecting a higher percentage of MR and CT exams.

The total combined salary cost for the three per diem diagnostic radiologists for this period was 38\% lower than the total compensation of one typical FTE body radiologist, while the total RVUs generated was only $8.3 \%$ lower than the typical FTE radiologist. Note that the per diem radiologists worked on a straight salary basis with no bonuses or benefits such as health insurance or pension plan contributions, which the regular university faculty received. However, the costs to the department for credentialing, staff dues, licensing fees and medical malpractice insurance per individual were the same in both groups.

\section{Value to pediatric radiologists}

Older radiologists, who compose approximately $7 \%$ of the radiology workforce [1], generally do not want to continue to work late or overnight shifts and would like to work fewer or no weekend days. Yet, if they have enjoyed their years of clinical practice, they might not be ready to give it up completely. These senior faculty members bring a wealth of experience and expertise to the table, which is an invaluable asset to their department and its more junior members. Bluth et al. [1] asserted that "[T] hey contribute expertise, judgment, and leadership and influence culture of the radiology practice environment." They are thus an asset to their department and institution. A summary of the advantages of having per diem senior pediatric radiologists is found in Table 2.
Table 2 Advantages of senior per diem pediatric radiologists

1. Clinical coverage

a. During short-term staff shortages, including high-demand vacation times

b. During periods of professional meetings

c. During a crisis (e.g., COVID-19), illness or unexpected emergencies

2. Scheduling flexibility

3. Expert and trusted clinical consultation

4. Experienced teaching (lectures and case collections)

5. Mentoring for research and career development

6. To allow fulfillment of required academic time for full-time colleagues

7. To allow full-time colleagues to focus on evolving technologies

8. Coverage of grant-required salary support

9. Contribution to morale and well-being

10. To enable gradual transition from full-time to complete retirement

11. Low cost

12. High reliability

COVID-19 novel coronavirus disease 2019

Donner et al. [3] explained that for the senor radiologist, "[B]ridge employment may play a significant role from fulltime employment to full-time retirement [and] allows for a gradual transition to a new and different life of socialization, relevance to the individual, and purpose to society." Offering a per diem option allows greater flexibility for the senior radiologist, thus satisfying his or her needs including a feeling of achievement, social contact, sense of identity, and income [3] while simultaneously giving the department greater depth and credibility. Harolds et al. [4] reviewed the advantages and disadvantages of part-time radiologist employment in great detail from the perspective of the individual as well as the institution and recommended that serious consideration be given to part-time options despite possible drawbacks. If proper attention is given to overcome potential obstacles, such a program can be a win-win situation.

From a workload perspective, every slot filled by a per diem person frees up a regular faculty member to pursue
Table 1 Productivity and compensation of three per diem vs. one full-time staff members during a 6-month period of Sept. 1, 2019, through Feb. 29, 2020

\begin{tabular}{lll}
\hline & 3 per diem staff & 1 full-time staff \\
\hline Number of days covered & 99 & 94 \\
Total number of DR studies reported & 4,034 & 3,918 \\
Total number of non-DR studies reported & 1,610 & 1,756 \\
Total number of studies reported & 5,644 & 5,674 \\
Relative value units (RVUs) & 2,052 & 2,238 \\
Percentage compensation & $100 \%$ & $138 \%$ \\
\hline
\end{tabular}

$D R$ digital radiography 
academic work, participate in professional meetings and fulfill administrative obligations. It allows for better coverage during times of national academic meetings and conferences, permitting higher participation in these activities. In our case, 99 days were covered by the per diem group, representing the equivalent of one FTE during that 6-month period.

In addition, senior radiologists generally take less time to read routine studies based on their experience and competence - they have seen it all before and have higher confidence levels, especially when it comes to radiographs and other conventional imaging [4]. They also provide an excellent go-to resource for younger faculty, who benefit from their experience and expertise. Menashe et al. [5] found that full-time academic pediatric radiologists have a favorable attitude toward part-time colleagues and value work-life balance and job satisfaction. Full-time faculty radiologists appreciate their senior colleagues' availability for questions of interpretation as well as general advice. Clinical colleagues also appreciate the availability and access to senior faculty they know and have confidence in. Senior radiologists are valuable mentors not only for clinical and educational activities, but also for research because most had significant research experience during the full-time phase of their academic careers.

\section{Value to the department and institution}

The impact of the per diem senior radiologist program on departmental research is not to be underestimated. In an academic pediatric radiology department with strong research emphasis, dedicated research time must be allocated. The ability to utilize the per diem senior radiologist to cover clinical work slots has made it possible to maintain a set percentage of academic time without the need to hire additional full-time faculty.

From a financial perspective, the per diem senior radiologist has thereby become the key in supporting research grants that involve salary support. A major challenge to the department when receiving a grant that includes salary support is how to manage coverage of the clinical duties of the grant recipient while the recipient fulfills the required research obligations. This is easily solved with engagement of per diem radiologists because it is almost impossible to hire someone new for a time-limited $20 \%$ position or even get a locum to cover just 1 day a week for a year.

During periods of unexpected crisis, such as the recent viral pandemic, per diem staff can be called upon to read cases from home and fill in for those who are either unable to work or are otherwise unavailable. They also can be called upon to conduct virtual teaching in as much as they have a wealth of preexisting material to draw from without much advance preparation.

Financially, the use of per diem employees, especially more than one, might obviate the need for hiring additional full-time faculty who (1) will cost more, (2) require more academic time to fulfill their career goals and (3) might not be readily available in a tight job market [6]. The per diem salary offered should be fair and sufficient to meet individual needs and not be a financial burden to the department. To determine reimbursement rates, market research should be done based on location and workload to assure that rates will be fair, competitive and attractive. Bonuses and benefits are high line-item costs and have a significant impact on the bottom line. Seniors who have officially retired from their university positions are very likely to not require these benefits because their health care and other benefits are already provided elsewhere, thereby further reducing their cost. In fact, the revenue generated by per diem staff might actually exceed the cost of the program. The costs of administration, licensing and credentialing, malpractice and hospital fees remain but should not be significant deterrents if taken into account. To date, we have not determined the number of days per year an individual per diem must work for the institution to break even financially.

In our situation the per diem radiologists became eligible for this program when they fully retired voluntarily from their university faculty positions and were invited by the department chair to participate. Our institution has an academic affiliation with, but is separate from, the university. A university faculty member can thus fully retire, achieving emeritus status, and subsequently apply (if invited) to become an employee of the hospital, which is not owned or operated by the university. When faculty members retire from the university and meet certain criteria, they become eligible for a retirement benefit package that is totally independent of future hospital employment.

There is also a difference between part-time faculty and per diem employment. Part-time university faculty usually receive pro-rated benefits such as health insurance, academic funds and academic time, not to mention participation in a bonus system and pension benefit, all of which cost the department. Nevertheless, they might participate in the call schedule and rotate through all modalities. However, some chairs believe that part-time faculty actually cost more than they produce, given these overhead costs. But per diem radiologists do not receive such additional benefits and are therefore less costly. Our data (Table 1) show that the per diem group of radiologists covered more day slots and read a similar number of examinations, albeit they were more heavily weighted toward lower RVU-producing examinations. Nevertheless, the number of RVUs generated by the per diems was only $8.3 \%$ less. The salary cost to the department was $38 \%$ lower than the salary of one FTE because it did not include the costs of bonuses and benefits, which were considerable. So there was no additional financial burden on the department. In fact, the per diems more than paid for their cost to the department. 


\section{Program drawbacks}

Nevertheless, several drawbacks of the per diem program should also be mentioned. Primarily, the main disadvantage of per diem staff from the department's perspective is the lack of coverage during weekend, holiday, late and overnight shifts. In the era where $24 / 7$ in-house attending coverage is becoming the norm, the need to have as many full-time staff available as possible to share these duties is obvious. Because we are a large department, overnight call coverage has not been an issue. If the per diems had ceased to work completely, additional staff might have been hired, but the impact on call frequency was not significant. The advantages of full-time staff are summarized in Table 3.

While the steps might be relatively easy for senior radiologists who are already in the organization wishing to transition to per diem status, the tasks associated with initial licensure and credentialing can be more daunting for senior radiologists who come from outside the system or state. For one of the authors, the process involved applying for a new medical license in two adjacent states, applying for employment at the new institution, and credentialing at that facility and four other hospitals serviced by the department of radiology. From time of onset to the first day of work, the process required a total of $50 \mathrm{~h}$ to complete and included drug tests, fingerprinting and completion of multiple redundant forms, all in duplicate or triplicate. But in our and in similar-size organizations there are dedicated staff to guide and support the radiologist to get through this tedious but necessary process. And so, despite the inconvenience and time-consuming paperwork, credentialing and licensing per diem faculty from out-of-state institutions can be achieved in the same manner as other new hires.

From a scheduling perspective, it is important that sufficient advance notice be given as to when additional coverage is needed and when per diem radiologists are available. So far, this has not been problematic as long as all parties can be flexible and reasonable. Balancing staffing requirements is highly important and must be given top priority. In most academic radiology settings, the "shift" concept of providing

Table 3 Advantages of a full-time pediatric radiologist compared to a senior per diem
1. Coverage of weekday call
2. Coverage of weekend call
3. Coverage of overnight in-house service
4. Coverage of all modalities
5. Mandatory participation in teaching
6. Research productivity
7. Administrative duties

service has predominated, so that continuity of care by single individuals is unnecessary and irrelevant. Flexibility in case of emergency situations is also advantageous.

We have not addressed the issue of aging-physician competency, which is a controversial topic. Weinreb [7] recently commented on stereotyping and discrimination on the basis of age and referenced sources opining that "competence, not age, determines the ability to practice medicine." Of course, incompetence can occur at any age. Our staff covers multiple hospital systems, and one of these does require a cognitive competency test for staff physicians older than 70 . It is a relatively simple computerbased examination that has nothing at all to do with medicine or radiology. All staff must maintain active licenses to practice medicine and their clinical privileges must be approved by the department chair. Participation in the Maintenance of Certification program of the American Board of Radiology is strongly encouraged and reimbursed. The per diem staff members participate in the same quality-improvement and internal peer-review programs as everyone else. Whether an individual is invited to participate or continue in the program is at the discretion of the department chair.

Of course, every department and academic institution is different. Changes to the traditional employment arrangements might meet resistance or be contrary to longestablished policy. Approval by the overseeing hospital legal and financial entities is necessary. Existing departmental and hospital policies and practice by-laws might need revision as well. Nevertheless, the benefits of retaining valuable senior members of the medical staff might outweigh arguments such as "we have never done it this way before" or "it's too difficult to arrange." Often, precedents for a per diem option exist and apply to nonphysician employees and can be cited.

Continuing medical education and mandatory institutional learning requirements for all practicing faculty still need to be met. The cost of that might require further negotiation and discussion. Other perks including the cost of journals, society memberships, office space, etc., also need to be negotiated and agreed upon, but the overall needs of the senor radiologist are usually less. As discussed by Harolds et al. [4], there are many factors to be considered but these need not be prohibitive.

\section{Summary}

In our experience, the development and institution of a per diem program to retain senior faculty at a large academic pediatric radiology department has benefited both individual senior faculty members as well as the radiology department, its full-time faculty, the hospital and, ultimately, patients (Table 2). It should be considered as an alternative 
bridge to total retirement and should be constructed to the mutual needs of the per diem faculty and the department to achieve a win-win result. Based on the current trends to promote wellness and prevent burnout, as well as to overcome a shortage of trained pediatric radiologists, this option might become the new norm for senior academic radiologists who still have much to offer and do not want to completely stop practicing or teaching. It permits more junior faculty members greater opportunity to achieve their academic goals by helping to reduce their clinical workload without additional cost to their departments.

Acknowledgments We thank Michael Francavilla and Parvez Kazmi for data collection and Soorosh Mahboubi for program participation.

\section{Compliance with ethical standards}

Conflicts of interest None

\section{References}

1. Bluth EI, Goodman RT, Bender CE (2018) The late-career radiologist: options and opportunities. Radiographics 38:1617-1625

2. Pfeifer CM (2018) Declining interest in pediatric radiology prompts a call to action. J Am Coll Radiol 15:490-492

3. Donner EM 3rd, Sze G, Bluth EI (2015) Retirement issues for radiologists: consensus statement on successful planning by the Commission on Human Resources of the ACR. J Am Coll Radiol 12:235-238

4. Harolds JA, Coleman BG, Recht MP et al (2014) The advantages, disadvantages, and policies for part-time radiologists: report of the ACR Commission on Human Resources. J Am Coll Radiol 11:668672

5. Menashe SJ, Paris MT, Chapman T et al (2018) Part-time pediatric radiology: the realities and perceptions of part-time employment in the academic setting. AJR Am J Roentgenol 211:971-977

6. Moriarity AK, Brown ML, Schultz LR (2014) Work and retirement preferences of practicing radiologists as a predictor of workforce needs. Acad Radiol 21:1067-1071

7. Weinreb J (2020) Musings on ageism and radiology. J Am Coll Radiol 17:1073-1074

Publisher's note Springer Nature remains neutral with regard to jurisdictional claims in published maps and institutional affiliations. 